TI 2015-118/III

Tinbergen Institute Discussion Paper

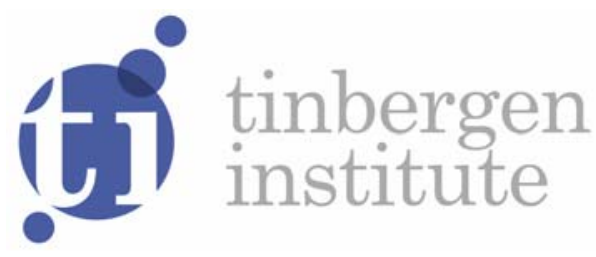

\title{
Exploiting Spillovers to forecast Crashes
}

\author{
Francine Gresnigt \\ Erik Kole \\ Philip Hans Franses
}

Erasmus School of Economics, Erasmus University Rotterdam, and Tinbergen Institute, the Netherlands. 
Tinbergen Institute is the graduate school and research institute in economics of Erasmus University Rotterdam, the University of Amsterdam and VU University Amsterdam.

More TI discussion papers can be downloaded at http://www.tinbergen.nl

Tinbergen Institute has two locations:

Tinbergen Institute Amsterdam

Gustav Mahlerplein 117

1082 MS Amsterdam

The Netherlands

Tel.: +31(0)20525 1600

Tinbergen Institute Rotterdam

Burg. Oudlaan 50

3062 PA Rotterdam

The Netherlands

Tel.: +31(0)10 4088900

Fax: +31(0)10 4089031 


\title{
Exploiting spillovers to forecast crashes *
}

\author{
Francine Gresnigt ${ }^{\dagger} \quad$ Erik Kole $\quad$ Philip Hans Franses \\ Econometric Institute, Erasmus School of Economics, Erasmus University Rotterdam \\ Tinbergen Institute, Erasmus University Rotterdam
}

October 21, 2015

\begin{abstract}
We develop Hawkes models in which events are triggered through self as well as crossexcitation. We examine whether incorporating cross-excitation improves the forecasts of extremes in asset returns compared to only self-excitation. The models are applied to US stocks, bonds and dollar exchange rates. In-sample, a Lagrange Multiplier test indicates the existence of cross-excitation for these series. Out-of-sample, we find that the models that include spillover effects forecast crashes and the Value-at-Risk significantly more accurately than the models without.
\end{abstract}

J.E.L. Classification: G17, G01

Key words: Hawkes processes, extremal dependence, Value-at-Risk, financial crashes, spillover

*All authors are from the Erasmus University Rotterdam. Francine Gresnigt is a PhD student at the Tinbergen Institute. Erik Kole is an associate research fellow and Philip Hans Franses is a research fellow at the Tinbergen Institute.

${ }^{\dagger}$ Corresponding author: Address: Burg. Oudlaan 50, Room H8-28, P.O. Box 1738, 3000DR Rotterdam, The Netherlands, Tel. +31 1040887 36. E-mail addresses: gresnigt@ese.eur.nl (Gresnigt), kole@ese.eur.nl (Kole) and franses@ese.eur.nl (Franses). 


\section{Introduction}

We develop Hawkes models in which events are triggered through self as well as cross-excitement. Exploiting the cross-sectional dependence between financial series, we aim to improve the forecasts of extremes and their sizes. It has already been shown that Hawkes models contribute to the identification and prediction of extreme events in financial markets. ${ }^{1}$ Currently, research in finance pays much attention to the modeling of extremal dependence between financial markets, though with an in-sample focus. We extend these studies on contagion, as we examine whether incorporating this dependence improves forecasts. For a wide range of assets, we find that Hawkes models with spillover effects forecast the probability of crashes and the Value-at-Risk significantly more accurately than models without.

Traders, regulators of financial markets and risk management benefit greatly from the accurate forecasts of extreme price movements in financial markets. Nowadays, a large literature focuses on modeling extremal dependence between financial markets. ${ }^{2}$ This topic gained interest since the financial crisis of 2008 as this crisis demonstrated the consequences of the cohesion between the behavior of the prices in the financial markets. For example on September 29, October 15 and December 1 in 2008 the S\&P 500, the Dow Jones Industrial Average (DJI) and the NASDAQ, all suffered top 20 percentage losses. Furthermore on September 29 the euro/dollar rate and the pound/dollar rate also dropped by a large amount, while the US bond market boomed. On the 16th of October, just one day after the major US stock markets crashed, and on the 1th of December both currencies fell again sharply. Moreover 4 days after these dates US bond prices shifted significantly. These joint extremes demonstrate the overlap of periods in which financial markets are subject to tension with extreme price movements as a result.

A Hawkes model uses an inhomogeneous Poisson process to model the occurrence of events above a certain threshold. The event rate increases with the arrival of a new event whereas the event rate decays with the time passed since an event. As the probability of events increases when an event occurs, the Hawkes process is called a self-exciting process. Characteristics typically observed in processes that fit Hawkes models are the clustering of extremes and serial dependence.

Earthquakes, for which the Hawkes models were originally designed, exhibit clustering behaviour in space as well as in time (Ogata, 1998). Likewise, financial series seem to cluster in a

\footnotetext{
${ }^{1}$ See amongst others: Bowsher, 2002, Chavez-Demoulin et al., 2005, Hewlett, 2006, Bowsher, 2007, Bauwens and Hautsch, 2009, Herrera and Schipp, 2009, Embrechts et al., 2011, Chavez-Demoulin and McGill, 2012, Bormetti et al., 2013, Aït-Sahalia et al., 2014, Gourieroux et al., 2014, Grothe et al., 2014. Aït-Sahalia et al., 2015, Gresnigt et al. $2015 \mathrm{a}$

${ }^{2}$ See amongst others: Longin and Solnik, 2001, Poon et al., 2003, Poon et al., 2004, Bekaert et al., 2011, Grothe et al., 2014., Aït-Sahalia et al., 2015
} 
dimension other than the time dimension. ${ }^{3}$ Extreme stock returns across markets are found to be more correlated than small returns (Bae et al., 2003). They occur more frequently at the same time than expected under the assumption of a normal dependence structure (Mashal and Zeevi, 2002, Hartmann, 2004, Sun et al., 2009). This suggests that financial markets experience stress at the same time. For example, using the multivariate GARCH framework, volatility spillover effects between stock markets have been detected in numerous studies. ${ }^{4}$ Interpreting volatility as a measure for the tension, these findings indicate that stress from financial markets pours over to other financial markets.

In this paper we extend Hawkes models to include contagion in financial markets. In the models we allow extreme events in one financial market to trigger both the occurrence and the magnitude of extreme events in other markets. Studies on Hawkes models for financial series in a multivariate setting include Bormetti et al. (2013), Grothe et al. (2014) and Aït-Sahalia et al. (2015). Different from these and other in-sample studies on financial contagion, we explicitly examine the effects of cross-excitation on out-of-sample forecasts. We assess its added value for the probability forecast of an extreme event and for Value-at-Risk. Thereby we extend Chavez-Demoulin et al. (2005), Herrera and Schipp (2009) and Chavez-Demoulin and McGill (2012), who showed using Value-at-Risk and Expected Shortfall that univariate Hawkes models contribute to the modeling and prediction of risk in finance. Moreover Byström (2004) finds that conditional models based on Extreme Value Theory give particularly accurate VaR measures, which are superior to traditional approaches such as GARCH for both standard and more extreme VaR quantiles.

In somewhat more detail, we use the Lagrange Multiplier principle (see Breusch and Pagan (1980), Engle (1982) and Hamilton (1996)) as in Gresnigt at al. (2015b) to test whether the spillovers contribute to the model fit. The correctness of the model specifications is further evaluated by means of the residual analysis methods as proposed in Ogata (1988). We assess the quality of the probability forecasts by the Quadratic and Log Probability Scores and the adjusted Mean Squared Error test of Clark and West (2007). For the evaluation of the VaR forecasts we use the unconditional coverage, independence and conditional coverage test of Christoffersen (1998), a Dynamic Quantile test in the line of Engle and Manganelli (2004) and the test designed by Diebold and Mariano (1995) based on an asymmetric loss function as in Giacomini and Komunjer (2005).

We apply the models to extreme losses in three stock markets and to extreme losses and gains in the US bond market and two exchange rates. Hence, for the stock market our analysis focuses on long positions, whereas we consider both long and short positions for bond and FX markets.

\footnotetext{
${ }^{3}$ See amongst others: Eun and Shim, 1989, Fischer and Palasvirta, 1990, King and Wadhwani, 1990, Lin et al., 1994, Connolly and Wang, 2003

${ }^{4}$ See amongst others: Hamao et al., 1990, Bae et al., 1994, Koutmos and Booth, 1995, Booth et al., 1997, Kanas, 1998.
} 
In-sample, the LM test reject the absence of cross-excitation. Performing residual analysis, the fit of the models for the various series is good, except for the bond market. Out-of-sample, models with spillover effects provide significantly more accurate forecasts of the occurrence of an extreme return and of the Value-at-Risk than the models without spillover effects. We conclude that including cross-sectional dependence improves the forecasts of crashes, and hence cross-sectional dependence should not be ignored. Furthermore, the predictability of event sizes adds to the forecasting performance of the models.

The rest of our paper is organized as follows. In Section 2 we give a brief introduction on Hawkes models. Furthermore we propose an extension of the univariate Hawkes model which incorporates cross-excitation and we discuss a Lagrange Multiplier test for dependence across series. In section 3 we apply the models and the LM test to US stocks, bonds and exchange rate data. Section 4 contains an in-sample assessment of the models by means of a residual analysis. The models are evaluated out-of-sample on the basis of the prediction of the probability of an extreme and the Value-at-Risk in Section 5. Section 6 concludes.

\section{Hawkes models}

\subsection{Univariate model}

The Hawkes model is a branching model. Each event can trigger subsequent events, and these can again trigger subsequent events. The model is based on the mutually self-exciting Hawkes point process, which is an inhomogeneous Poisson process. For the Hawkes process, the intensity at which events arrive at time $t$ depends on the history of events prior to time $t$.

Consider an event process $\left(t_{1}, m_{1}\right), \ldots,\left(t_{N}, m_{N}\right)$ where $t_{i}$ defines the time and $m_{i}$ the mark of event $i$. Let $\mathcal{H}_{t}=\left\{\left(t_{i}, m_{i}\right): t_{i}<t\right\}$ represent the entire history of events up to time $t$. The conditional intensity of jump arrivals following a Hawkes process is represented by

$$
\lambda\left(t \mid \boldsymbol{\theta} ; \mathcal{H}_{t}\right)=\mu+\gamma_{1} \sum_{i: t_{i}<t} g\left(t-t_{i}, m_{i}\right),
$$

where $\mu>0, \gamma_{0}>0$ and $g(s, m)>0$ whenever $s, m>0$ and 0 elsewhere. The parameter $\gamma_{1}$ controls the maximum triggering intensity and the expected number of events directly triggered by an event. The conditional intensity consists of a constant term $\mu$ and a self-exciting function $\gamma_{1} g(s, m)$, the latter function depends on the time passed since the jumps which occurred before $t$ and the size of these jumps. Thus the rate at which events arrive is separated in a long-term background component and a short-term clustering component describing the temporal distribution of aftershocks. The conditional intensity uniquely determines the distribution of the process. 
It is usual to specify the triggering function as

$$
g(s, m)=e^{-\beta s} c(m)
$$

where $\beta$ determines how fast the possibility of triggering events decays depending on the time passed since an event. The influence of the sizes of past events on the intensity is given by $c(m)$.

For the influence of the sizes of past events on triggering of future events, $c(m)$, it is common to use the exponential form

$$
c(m)=e^{\alpha(m-u)}
$$

where $u$ represents the minimum magnitude of an event and $\alpha$ determines how the size of an event affects the probability of triggering other events. When $\alpha>0$, larger events trigger more events than smaller events, because the probability of triggering events increases with the size of past events $(m)$. The larger positive $\alpha$ is, the more pronounced is the influence of the size of events.

To enable an application to financial data, we choose a Generalized Pareto Distribution for the sizes of the events ${ }^{5}$

$$
\operatorname{Pr}\left(m \leq M \mid m \geq u ; \boldsymbol{\theta} ; \mathcal{H}_{t}\right)=1-\left(1+\xi \frac{m-u}{\sigma(t)}\right)^{-1 / \xi}
$$

where $\sigma(t)=\phi+\frac{\eta}{\gamma_{1}} \lambda\left(t \mid \boldsymbol{\theta} ; \mathcal{H}_{t}\right)$ and $\xi \neq 0$. In case $\eta=0$ the size of an event is unpredictable, whereas in case $\eta \neq 0$ the arrival times and sizes of previous events affect the distribution of the sizes of subsequent events. We scale the mean and variance of the distribution of the sizes of events with $\sigma(t)$. When $\eta>0$ the magnitude of events is expected to be more extreme, when the conditional intensity is high. The larger positive $\eta$ is, the more pronounced is the influence of the history of events on the size of subsequent events.

The expected number of off-spring of an event, is given by the branching ratio. Using (2) the branching ratio is equal to

$$
\gamma_{1} \int_{0}^{\infty} g(s, m) d s=\frac{\gamma_{1}}{\beta} c(m)
$$

A Hawkes process is stationary when the branching ratio is smaller than 1 . When the ratio exceeds 1 , the rate at which events arrive will grow to infinity over time.

The log-likelihood of the Hawkes model, specified in the conditional intensity and the probability distribution of the sizes of the events, is given by

$$
\log L(\boldsymbol{\theta})=\sum_{i=1}^{N} \log \lambda\left(t_{i} \mid \boldsymbol{\theta} ; \mathcal{H}_{t}\right)-\int_{0}^{T} \lambda\left(s \mid \boldsymbol{\theta} ; \mathcal{H}_{s}\right) d s+\sum_{i=1}^{N} \log f\left(m_{i} \mid \boldsymbol{\theta} ; \mathcal{H}_{t}\right)
$$

\footnotetext{
${ }^{5}$ See amongst others: Poon et al., 2004, Herrera and Schipp, 2009, Chavez-Demoulin et al., 2005, and ChavezDemoulin and McGill ,2012, Grothe et al., 2014, Gresnigt et al., 2015a and b
} 
where $t_{i}$ are the event arrival times in the interval $[0, T]$.

The probability of the occurrence of an event following a Hawkes process with conditional intensity $\lambda\left(t \mid \boldsymbol{\theta} ; \mathcal{H}_{t}\right)$ between $t-\delta t$ and $t$ is now given by

$$
\begin{aligned}
\operatorname{Pr}\left(N(t)-N(t-\delta t)>0 \mid \boldsymbol{\theta} ; \mathcal{H}_{t}\right) & =1-\operatorname{Pr}\left(N(t)-N(t-\delta t)=0 \mid \boldsymbol{\theta} ; \mathcal{H}_{t}\right) \\
& =1-F\left(t^{*}>\delta t \mid \boldsymbol{\theta} ; \mathcal{H}_{t}\right) \\
& =\exp \left(-\int_{t-\delta t}^{t} \lambda\left(s \mid \boldsymbol{\theta} ; \mathcal{H}_{s}\right) d s\right)
\end{aligned}
$$

We follow Gonzalo and Olmo (2004) to determine the threshold above which an event is extreme. The selection of the threshold is important as a too low threshold gives inaccurate parameter estimates. On the other hand, a threshold higher than necessary reduces the number of observations available to estimate the model, resulting in estimates that are inefficient and more sensitive to aberrant observations. Gonzalo and Olmo (2004) define the weighted Picklands distance as the maximum distance between the empirical distribution of observations above a certain threshold and the maximum likelihood fit of the General Pareto Distribution to these observations multiplied by a tuning parameter,

$$
d^{W P}\left(F_{u, N}, G P D_{\hat{\xi}_{N}^{M L}(u), \hat{\sigma}_{N}^{M L}(u)}\right)=k^{\epsilon} \sup _{0 \leq y<\infty}\left|F_{u, N}(y)-G P D_{\hat{\xi}_{N}^{M L}(u), \hat{\sigma}_{N}^{M L}(u)}(y)\right|,
$$

where $0 \leq \epsilon \leq \frac{1}{2}$ and $k=\sum_{i=1}^{N} \mathrm{I}\left(y_{i}>u\right)$. The parameter $\epsilon$ determines the weight assigned by $d^{W P}$ to the tail observations defined by the corresponding $u$. When $\epsilon=0$, the distance is equal to the distance used by Pickands (1975). When $\epsilon=\frac{1}{2}$ the distance is equal to the KolmogorovSmirnov (KS) statistic. The optimal threshold minimizes this distance

$$
u_{N}^{*}=\arg \min _{u} d^{W P}\left(F_{u, N}, G P D_{\hat{\xi}_{N}^{M L}(u), \hat{\sigma}_{N}^{M L}(u)}\right)
$$

Following Gonzalo and Olmo (2004), we set $\epsilon=\frac{1}{2}$. Other techniques for setting $u_{N}^{*}$ have their drawbacks. Graphical methods, such as the interpretation of mean-excess, threshold stability and Hill plots, are ambiguous and depend on choices in earlier stages, which leads to subjective results. Besides, these methods are time consuming. Block maxima methods are in general not applicable to financial series as the anti-clustering condition is violated for those series. Many bootstrap approaches set the threshold on the basis of biased parameter estimates. For a more extensive discussion on threshold estimation see Scarrott and MacDonald (2012).

\subsection{Spillover model}

When events across series tend to arrive around the same time, the occurrence of an event in one series could increase the probability that an event in another series arrives. A Hawkes model in 
which the conditional intensity of a series $i$ is affected by the history of the event process of another series $j$ is represented as

$$
\lambda\left(t \mid \boldsymbol{\theta} ; \mathcal{H}_{t}\right)=\mu+\gamma_{1} \sum_{i: t_{i}<t} g\left(t-t_{i}, m_{i}\right)+\gamma_{2} \sum_{j: t_{j}<t} h\left(t-t_{j}, m_{j}\right),
$$

where $\gamma_{2} h\left(t-t_{j}, m_{j}\right)$ presents the effect of an event in series $j$ on the intensity at time $t$ in series $i$. The cross-exciting function $h(s, m)$ can be specified in various ways. Here, we set $h(s, m)=g(s, m)$ to keep the model parsimonious and model parameters identified when there are no spillovers, $\gamma_{2}=0$. The events in the series $j$ also affect the probability distributions of the sizes of events in series $i$ through (4) when $\eta \neq 0$.

We perform a Lagrange Multiplier test on the null-hypothesis that $\gamma_{2}=0$. As we deal with a count process we define the score used for the LM test in event numbers and not in time points. The score of the $n$-th event is defined as the derivative of the logarithm of the conditional likelihood (6) with respect to the parameter vector $\boldsymbol{\theta}$,

$$
\mathbf{g}_{i}(\overline{\boldsymbol{\theta}})=\left.\frac{\partial \log p\left(y_{i} \mid \boldsymbol{\theta} ; \mathcal{H}_{t}\right)}{\partial \boldsymbol{\theta}}\right|_{\boldsymbol{\theta}=\overline{\boldsymbol{\theta}}}
$$

which can be derived to be equal to

$$
\mathbf{g}_{i}(\tilde{\boldsymbol{\theta}})=-\frac{\partial \int_{t_{i-1}}^{t_{i}} \lambda\left(s \mid \boldsymbol{\theta} ; \mathcal{H}_{s}\right) d s}{\partial \boldsymbol{\theta}}+\left.\frac{1}{\lambda\left(t_{i} \mid \boldsymbol{\theta} ; \mathcal{H}_{t}\right)} \frac{\partial \lambda\left(t_{i} \mid \boldsymbol{\theta} ; \mathcal{H}_{t}\right)}{\partial \boldsymbol{\theta}}\right|_{\boldsymbol{\theta}=\tilde{\boldsymbol{\theta}}}+\left.\frac{\partial \log f\left(m_{i} \mid \boldsymbol{\theta} ; \mathcal{H}_{t}\right)}{\partial \boldsymbol{\theta}}\right|_{\boldsymbol{\theta}=\tilde{\boldsymbol{\theta}}}
$$

The score (12) consists of three terms that respectively reflect the change in the probability distribution of the time between two consecutive events, the relative change in the rate at which events occur and the change in probability to observe an event of a certain magnitude, when $\tilde{\boldsymbol{\theta}}$ is shifted by a infinitesimal amount. We assess the added value of the parameters to the model in terms of their contribution to the prediction of the occurrence times and magnitudes of events $t_{i}$ and $m_{i}$.

Let $x(t)=\sum_{j: t_{j}<t} h\left(t-t_{j}, m_{j}\right)$ denote the additional tension caused by all events in a series $j$ before time $t$. To test whether the cross-triggering effects are significant for a series $i$, we first estimate the Hawkes model under the null-hypothesis of no cross-excitation, $\gamma_{2}=0$. Next, we use (12) to compute the expected score from the derivative of the conditional intensity, the integrated conditional intensity and the probability distribution of event sizes, given by

$$
\begin{aligned}
\frac{\partial \lambda\left(t_{i} \mid \boldsymbol{\theta} ; \mathcal{H}_{t}\right)}{\partial \gamma_{2}} & =x\left(t_{i}\right) \\
\frac{\partial \int_{t_{i-1}}^{t_{i}} \lambda\left(s \mid \boldsymbol{\theta} ; \mathcal{H}_{s}\right) d s}{\partial \gamma_{2}} & =\int_{t_{i-1}}^{t_{i}} x(s) d s \\
\frac{\partial \log f\left(m_{i} \mid \boldsymbol{\theta} ; \mathcal{H}_{t}\right)}{\partial \gamma_{2}} & =\frac{\eta x\left(t_{i}\right)}{\gamma_{1} \sigma\left(t_{i}\right)}\left(\frac{\xi+\left(m_{i}-u_{i}\right)}{\sigma\left(t_{i}\right)+\xi\left(m_{i}-u_{i}\right)}-1\right) .
\end{aligned}
$$

The LM test is asymptotically $\chi^{2}(1)$ under the null of no cross-excitation. 


\section{Application to Financial Data}

We consider daily returns of three US stock market indices, the S\&P 500 (S\&P), the NASDAQ (NDQ) and the Dow Jones Industrial Average (DJI), the US bond index (BND), and two exchange rates, the euro/dollar rate $(€ / \$)$ and the pound/dollar rate $(£ / \$)$ over the period from 1 January, 1990, to 1 July, 2015. We construct daily discrete returns as $R_{t}=\left(p_{t} / p_{t-1}-1\right) \cdot 100$, where $p_{t}$ denotes the value of a series at $t$. The Hawkes models are estimated over the period that ranges from 1 January, 1990, to 1 January, 2013, which corresponds to 6653 trading days. We use the data thereafter for an out-of-sample evaluation of the models. Figure 1 displays the evolution of the prices of the stock market indices, the bond market index and the exchange rates over the whole period.

[Figure 1 about here.]

We apply the Hawkes models to negative extremes in the stock market indices and to negative and positive extremes in the US bond index and the exchange rates. Employing the method of Gonzalo and Olmo (2004) over the estimation period, we find that extremes above the $94.29 \%$ among all series can be considered to be 'really extreme'. We set the threshold for each series at the value that corresponds to this quantile of their empirical distribution.

The estimated Hawkes models differ as the parameters $\alpha, \eta$ and/or $\gamma_{2}$ are restricted to zero in some of the models, while in other models these parameters are unrestricted. When $\alpha=0$ the sizes of former events do not influence the conditional intensity of new event arrivals, whereas they do when $\alpha \neq 0$. When $\eta=0$ the history of the event process does not have information on the sizes of events in the future, while when $\eta \neq 0$ the sizes of events can be predicted from the past.

Our specific focus is on comparing the models in which $\gamma_{2}=0$ to the models in which $\gamma_{2} \neq 0$. In the first set of models the occurrence of events in a series and the magnitudes of these events are not affected by the occurrence of events in another series. In the second set of models the rate at which events arrive in a series $i$ is amplified whenever an event in another series $j$ arrives. The effect scales with the size of the events in the series $j$ whenever $\alpha \neq 0$. Moreover, in case $\eta \neq 0$ the occurrence of events in the series $j$ influences the distribution of the sizes of events in the series $i$. In this paper we examine the impact of crashes in the S\&P 500 index on extremes in the other series. We choose to investigate the spillovers from the S\&P 500 index as the index is one of the most commonly followed equity indices and considered as one of the best representations of the US stock market. Furthermore we investigate whether crashes in the NASDAQ provoke crashes in the S\&P 500 index. The NASDAQ is very well suited to serve as a proxy for spillover effects to the S\&P 500 index from other stock market indices as the NASDAQ is the second-largest exchange in the world by market capitalization. 
[Table 1 about here.]

Table 1 presents the parameter estimates for the Hawkes model in which $\gamma_{2} \neq 0, \eta \neq 0$ and $\alpha=0$. As $\gamma_{2} \neq 0$, crashes in the $\mathrm{S} \& \mathrm{P} 500$ index are allowed to provoke extremes in the other series, while crashes in the S\&P 500 index can be triggered by crashes in the NASDAQ. Due to space constraints we only display the parameter estimates for this model. This model turns out to be the overall best performing model out-of-sample.

The estimates for $\eta$ are positive and significant for all series. This suggests that a model which incorporates the history of the event process to predict the sizes of subsequent events, matches the extremes closer than a model which assumes that the sizes of events are independent of the past. As $\eta>0$, the size distribution has a higher mean and variance when the conditional intensity is high such that the magnitude of events is expected to be more extreme in that case. This means that, on average larger events are observed in turbulent periods in which more events occur than in tranquil periods. As $\alpha$ is restricted to zero, the sizes of previous crashes do not affect the rate at which new crashes occur.

The parameter $\gamma_{2}$ controls for the cross-triggering of events. As is clear from the bottom row of Table 1, all estimated parameters are significant at the $5 \%$ level except for the parameter that drives the effect of crashes in the S\&P 500 index on crashes in the NASDAQ. Moreover, the parameters linked to the spillovers from crashes in the S\&P 500 index to extremes in the exchange rates and crashes in the US bond market, are significant at a $1 \%$ level. The instantaneous effect of the occurrence of events in one series on the event rate in another series is between 0.15 and 0.62 times as large as the effect of events in the series itself, where the ratio between the instantaneous cross-excitation and self-excitation effect is the highest for extremes in the bond market and the lowest for crashes in the NASDAQ.

To quantify the effects that extremes occurring in one market have on another market, we examine the branching ratio of extremes in the markets, see (5). Amongst series, an event triggers on average 0.11 to 0.41 events in another series, where the smallest amount of crashes are provoked between the NASDAQ and the S\&P 500 index. The expected number of bond crashes following a S\&P 500 crash is the highest. The percentage of events induced by events in another series ranges between $12 \%$ and $34 \%$.

Looking at the parameter estimates, the impact of crashes in the S\&P 500 index on extremes in other series and the impact of crashes in the NASDAQ on crashes in the S\&P 500 index, seems quite substantial such that it should not be ignored. We now perform an LM test to confirm the indicated cross-excitation.

[Table 2 about here.] 
Table 2 reports the LM statistics for testing the null-hypothesis of no spillover effects $\gamma_{2}=0$ in various models. In accordance with the parameter estimates, we find that crashes in the S\&P 500 index amplify the probability that crashes as well as booms in the bond market and the exchange rate series occur. Thus, the LM test detects both stock-bond contagion as the flight-to-quality phenomenon, that is, the outflow of capital from stock markets to bond markets when the first are facing crises periods. The evidence for stock-bond contagion is more pronounced as the LM test statistics for the effect of crashes in the S\&P 500 to crashes US bond market are by far the highest, ranging from 33.98 to 34.51 .

Furthermore, as the parameter estimates already indicated, we discover that when Hawkes models are applied in which the sizes of the past crashes have no impact on the occurrence of future crashes, crashes in the S\&P 500 index trigger crashes in the DJI index. However, the LM test suggests that there is no such cross-excitement when models are employed in which sizes of previous crashes matter for the arrival rate of new crashes. Also, according to the LM test, there is a lack of spillover effects from the S\&P 500 index to the NASDAQ. We do encounter evidence for spillover effects the other way around. Both findings are reflected in the parameter estimates in Table 1.

From the parameter estimates and the LM tests, we conclude that in-sample the impact of the occurrence of crashes in the NASDAQ on the occurrence of crashes in the S\&P 500 index and the impact of the occurrence of crashes in the S\&P 500 index on the occurrence of extremes in other financial series is sizeable and important. We may thus expect that the Hawkes models that account for the cross-triggering phenomenon perform better out-of-sample than the Hawkes models that ignore it.

\section{Residual analysis}

We assess the goodness-of-fit of our models using the residual analysis technique of Ogata (1988). If the event process $\left\{t_{i}\right\}$ is generated by the conditional intensity $\lambda(t)$, the transformed times

$$
\tau_{i}=\int_{0}^{t_{i}} \lambda(s) d s
$$

are distributed according to a homogeneous Poisson process with intensity 1 . Furthermore the transformed interarrival times

$$
\tau_{i}-\tau_{i-1}=\int_{t_{i-1}}^{t_{i}} \lambda(s) d s
$$

are independent exponential random variables with mean 1 . If the models are correctly specified, $\lambda(t)$ can be approximated by $\lambda\left(t \mid \widehat{\theta} ; \mathcal{H}_{t}\right)$. The sequence $\left\{\tau_{i}\right\}$ is called the residual process. In order to verify whether the residual process derived from the models is Poisson with unit intensity, we 
perform the Kolmogorov-Smirnov (KS) test. The null-hypothesis of our test is that the distribution of the residual process and the unit Poisson distribution are equal.

[Table 3 about here.]

The KS tests are performed on the transformed times by applying the models to the extremes. The $p$-values of the tests are reported in Table 3. The $p$-values indicate that the extremes do not deviate from an event process specified by the models at a $5 \%$ level, except for the US bond crashes and booms when spillover effects from crashes in the S\&P 500 index are incorporated. Though $p$ values are higher for the models without cross-excitation, they cannot be interpreted as a preference for these models.

\section{Forecasting}

\subsection{Probability predictions}

Traders, risk managers and regulators of financial markets can benefit from the accurate forecast of a crash. The probability of an extreme event occurring between $t-\delta t$ and $t$ is given by (7). We evaluate the probability forecast of an extreme event during the next day in stock, bond and FX markets out-of-sample from 1 January, 2013, to 1 July, 2015. We use the in-sample thresholds and parameter estimates of the previous section to prevent any look-ahead bias.

To compare the probability forecasts, we compute the Quadratic Probability Score (QPS) and the Log Probability Score (LPS) for each model, that is

$$
\begin{aligned}
Q P S & =\frac{2}{T} \sum_{t=1}^{T}(\hat{p}(t)-\mathrm{I}(r(t)>u))^{2} \\
L P S & =-\frac{1}{T} \sum_{t=1}^{T}[(1-\mathrm{I}(r(t)>u)) \log (1-\hat{p}(t))+\mathrm{I}(r(t)>u) \log (\hat{p}(t))],
\end{aligned}
$$

where $t$ is a day and $T$ is the total number of days. Here $p_{t}$ represents the probability forecast of an extreme on day $t$, and $\mathrm{I}(r(t)>u)$ is an indicator function taking the value 1 when the return at time $t$ exceeds the threshold and the value 0 otherwise. The QPS and LPS range respectively from 0 to 1 and from 0 to $\infty$, with 0 indicating perfect accuracy. When the QPS or the LPS are higher, the probability forecasts deviate more from a binary variable indicating the occurrence of events. The LPS punishes large deviations heavier than small deviations.

[Table 4 about here.] 
The QPS and the LPS of the models for all series are displayed in Table 4. For all series, the QPS and LPS are lower when spillover effects are included in the models, except for booms in the bond market. The difference in scores between models with and without cross-excitation is most visible for the LPS.

With the adjusted Mean Squared Prediction Error (adjusted-MSPE) of Clark and West (2007) we check whether the probability forecasts of models with cross-excitation are significantly more accurate than models without. Under the null, the unrestricted model produces forecasts that contain more noise by estimating parameters which actually have the value zero. Therefore, the MSPE of the restricted model is expected to be smaller. To account for this noise, the MSPE needs to be adjusted. The test of Clark and West (2007) is one-sided, as the test only gives information about whether the unrestricted model is significantly more accurate than the rectricted one. The adjustedMSPE statistics comparing probability predictions of models with and without cross-excitation are given in Table 5. The adjusted-MSPE statistics comparing probability predictions of models with and without influence of the event sizes on the conditional intensity and models with and without influence of the history of the event process on the event sizes, are given in Table 6.

[Table 5 about here.]

From Table 5 we conclude that, the probability predictions of models with spillovers are significantly more accurate for the extremes examined at a 5\% level, except for booms in the bond market. The adjusted-MSPE statistics are especially high for negative extremes in the pound/dollar-rate. In line with the LM statistics and the parameter estimates, we find that the adjusted-MSPE statistics are the lowest among the positive statistics for crashes in the NASDAQ.

[Table 6 about here.]

According to the the adjusted-MSPE statistics in Table 6, the probability predictions of models with predictable event sizes are significantly more accurate for the extremes examined at a $5 \%$ level. The adjusted-MSPE statistics show that the incorporation of a triggering effect that depends on the size of events does not lead to more accurate probability predictions.

Our main result in this section is that, all in all, the Hawkes models that account for crosstriggering effects deliver more accurate forecasts of the occurrence of extremes in financial markets than the Hawkes models that ignore the existence of spillovers.

\subsection{Value-at-Risk}

To avoid large losses due to price changes and to meet regulatory requirements that limit exposure to risk, investors in financial markets use Value-at-Risk to quantify the maximum of returns 
associated with a certain confidence level. The $\operatorname{VaR}(t)$ is defined as the $\alpha$-th quantile of a distribution at time $t$ which is the solution to $\operatorname{Pr}\left(m \geq q \mid \boldsymbol{\theta} ; \mathcal{H}_{t}\right)=1-\alpha$. As $\operatorname{Pr}\left(m \geq q \mid \mathcal{H}_{t}\right)=$ $\operatorname{Pr}\left(N(t)-N(t-\delta t)>0 \mid \boldsymbol{\theta} ; \mathcal{H}_{t}\right) \times \operatorname{Pr}\left(m \geq q \mid m \geq u ; \boldsymbol{\theta} ; \mathcal{H}_{t}\right)$ which are given in (7) and (4), we have that

$$
\operatorname{VaR}_{\alpha}(t)=u+\frac{\sigma(t)}{\xi}\left(\left(\frac{1-\alpha}{1-\exp \left(-\int_{t-\delta t}^{t} \lambda\left(s \mid \boldsymbol{\theta} ; \mathcal{H}_{s}\right) d s\right)}\right)^{-\xi}-1\right)
$$

where $\sigma(t)=\phi+\frac{\eta}{\gamma_{1}} \lambda\left(t \mid \boldsymbol{\theta} ; \mathcal{H}_{t}\right)$ and $\lambda\left(t \mid \boldsymbol{\theta} ; \mathcal{H}_{t}\right)$ is given by (10).

[Figure 2 about here.]

Figure 2 shows the VaR predictions for the 95\% confidence level out-of-sample from 1 January, 2013 , to 1 July, 2015. From the graph we can conclude that the VaR predictions for the S\&P 500 index produced by the models are quite different. For these series the Value-at-Risk for the model without cross-excitation $\left(\gamma_{2} \neq 0\right)$ and with predictable event sizes $(\eta \neq 0)$ being the highest followed (i) by the model without restrictions, (ii) the model in which both parameters are restricted and (iii) the model in which $\gamma_{2} \neq 0$ and $\eta=0$, over almost the entire forecast period. The predictions of the models with cross-excitation vary more over time than the predictions of the models without cross-excitation. For the two stock indices both the VaR predictions of the models with unpredictable event sizes and the VaR predictions of the models with unpredictable event sizes are close in value over time, where the value of the first is much higher than that of the second. The VaR predictions for the bond market and the exchange rates behave similarly. For these series, the same ranking as for the S\&P 500 index applies.

We evaluate the VaR forecasts (20) by comparing the estimated conditional VaR for one day time horizon, that is $\delta t=1$, for the confidence levels $\alpha=\{0.95,0.99\}$ with the actual returns. To quantify the quality of the VaR forecasts we use the unconditional coverage $\left(L R_{u c}\right)$ test, the independence ( $\left.L R_{i n d}\right)$ test and the conditional coverage $\left(L R_{c c}\right)$ test of Christoffersen (1998) and a Dynamic Quantile (DQ) test in the line of Engle and Manganelli (2004). Furthermore, to compare the VaR forecasts of different models, we define an asymmetric loss function as Giacomini and Komunjer (2005) and test whether the loss differentials are significant using the test designed by Diebold and Mariano (1995) (DM test).

With the unconditional coverage $\left(L R_{u c}\right)$ test we check whether the actual fraction of VaRviolations equals the theoretical proportion of $1-\alpha$. We let $x_{\alpha}(t)$ denote an indicator function taking the value 1 when the extreme is larger than the $\operatorname{VaR}_{\alpha}(t)$ and the value 0 otherwise. If the models are correct $\mathrm{E}\left[x_{\alpha}(t)\right]=1-\alpha$. The independence $\left(L R_{\text {ind }}\right)$ test concerns independence among the VaR-violations such that a violation today has no influence on the probability of a 
violation tomorrow. A combination of the $L R_{u c}$ and $L R_{\text {ind }}$ is given by the conditional coverage $\left(L R_{c c}\right)$ test. The $L R_{u c}, L R_{\text {ind }}$ and $L R_{c c}$ test statistics for the confidence levels $95 \%$ and $99 \%$ are given in Table 7 and Table 8, respectively.

[Table 7 about here.]

[Table 8 about here.]

Table 7 shows that for the $95 \%$ confidence level, the unconditional and thereby conditional coverage of the VaR predictions is a problem whenever the history of the event process has no influence on the sizes of subsequent events $(\eta=0)$. The models that include the predictability of event sizes $(\eta \neq 0)$ and cross-excitation $\left(\gamma_{2} \neq 0\right)$ lead to the lowest statistics. While the effect of past events on the size is largest, allowing for cross-excitation leads to a further improvement. The effect of the size of events on the triggering intensity (the parameter $\alpha$ ) has only a small effect. The model with cross-excitation effects $\left(\gamma_{2} \neq 0\right)$ and unpredictable event sizes $(\eta=0)$ fails the unconditional and conditional coverage tests at a 5\% level for almost all series. The same model without cross-excitation effects fails the tests for more than half of the series. The unconditional and conditional coverage test statistics are especially high for the NASDAQ. Moreover, in case $\eta=0$, the Hawkes models also do not pass the independence test at a 5\% level for this series. The VaR-violations are independent for all other series. When event sizes are predictable $(\eta \neq 0)$, the fraction of VaR-violations does not significantly deviate from the theoretical $5 \%$ at a $5 \%$ level for all series except for the S\&P 500 index. Furthermore, the VaR-violations do not suffer from dependence such that the models pass the conditional coverage test except for the S\&P 500 index. The test statistics for the VaR predictions at the 99\% confidence level, reported in Table 8, are, with only a few exceptions, insignificant.

Besides the independence test of Christoffersen (1998), we use the Dynamic Quantile (DQ) test of Engle and Manganelli (2004) to verify whether VaR-violations are predictable from the history of the event process. If the models are correct, VaR-violations before time $t$ should have no explanatory power for a VaR-violation at $t$. The DQ test is implemented as in Berkowitz et al. (2011), that is the errors $\epsilon(t)$ from the regression

$$
x_{\alpha}(t)=\beta_{0}+\beta_{1} x_{\alpha}(t-1)+\epsilon(t)
$$

follow a logistic distribution. In this way we account for heteroscedasticity due to the binary nature of the variable $x_{\alpha}(t)$. The null hypothesis that $\beta_{1}=0$ is tested with a Likelihood Ratio test.

[Table 9 about here.] 
The DQ test statistics for the VaR predictions at the confidence levels $95 \%$ and $99 \%$ are given in Table 9. Almost all test statistics are insignificant. In accordance with the independence test of Christoffersen (1998), the DQ test indicates that the models in which event sizes are unpredictable fail to employ all information in the history of the event process to produce VaR predictions at the confidence levels $95 \%$.

To compare the VaR predictions made by the different models we use the test of Diebold and Mariano (1995) (DM test) based on the asymmetric tick loss function of Giacomini and Komunjer (2005)

$$
\mathrm{L}\left(e_{\alpha}^{k}(t)\right)=\left(\alpha-\mathrm{I}\left(e_{\alpha}^{k}(t)<0\right)\right) e_{\alpha}^{k}(t)
$$

where $e_{\alpha}^{k}(t)=r(t)-\left(-V a R_{\alpha}^{k}(t)\right)$ is the difference between the actual return and the predicted VaR at confidence level $\alpha$ and $\mathrm{I}\left(e_{\alpha}^{k}(t)<0\right)$ is an indicator function which takes the value 1 when the negative of the return at time $t$ is larger than the predicted $\mathrm{VaR}$ at confidence level $\alpha$ and the value 0 otherwise. As the loss function (22) is asymmetric, realized returns below the negative of $\operatorname{VaR} R_{\alpha}^{k}(t)$ lead to larger losses.

[Table 10 about here.]

The DM statistics comparing VaR predictions of models with and without cross-excitation at the confidence levels $95 \%$ and $99 \%$ are given in Table 10. From the DM tests we conclude that, at the $95 \%$ confidence level, the models with cross-excitation deliver significantly more accurate VaR predictions for investors with a long position in the stock markets, the bond market and the exchange rates and investors with a short position in the exchange rates. Moreover, these investors should again employ the models with cross-excitation for their VaR predictions at the 99\% confidence level when the event sizes in the models are unpredictable, as these models produce significantly more accurate VaR predictions than the models without cross-excitation. For the investors with a long position in the S\&P 500 index, VaR predictions of the models with crossexcitation are also significantly more accurate when the event sizes in the models are predictable. For other investors, VaR predictions are less accurate. Investors with a short position in the bond market should only employ models with cross-excitation when the event sizes in the models are predictable and when they are interested in predicting the VaR at the $95 \%$ confidence level.

[Table 11 about here.]

The DM statistics comparing probability predictions of models with and without influence of the event sizes on the conditional intensity and models with and without influence of the history of the event process on the event sizes, at the confidence levels $95 \%$ and $99 \%$ are given in Table 11. According to the DM tests, using models with influence of the history of the event process on 
the event sizes leads to significantly more accurate VaR predictions in almost all cases. Only the VaR predictions for the S\&P 500 index at the $99 \%$ confidence level are significantly less accurate. For the incorporation of the influence of the event sizes on the conditional intensity, the results are mixed. Roughly, investors with a long position in the NASDAQ, the Dow Jones Industrial Average and investors with a short position in the bond market could benefit from employing these models for their VaR predictions, while investors with a long position in the bond market and the euro/dollar rate and investors with a short position in the exchange rates do better employing models without influence of the event sizes on the conditional intensity.

Our key finding is that, all in all, the models that account for cross-triggering provide better VaR predictions, especially at the $95 \%$ confidence level. This is not surprising as these models are also ranked the highest in case the predictions of the probability of an extreme are evaluated. Therefore we conclude that traders, regulators of financial markets and risk management should incorporate spillovers in their models when they are employing Hawkes models to forecast extremes in financial markets. Also the predictability of event sizes adds to the model performance, whereas incorporation of the influence of the event sizes on the conditional intensity delivers mixed results.

\section{Conclusion}

We extend Hawkes models to account for cross-excitation between financial markets. We assess the contribution of including cross-excitation for forecasting extreme events and the Value-at-Risk. We apply the models to extreme negative returns in the S\&P 500 index, the NASDAQ and the Dow Jones Industrial Average, and to extreme negative and positive returns in the US bond index, the euro/dollar rate and the pond/dollar rate over the period from 1 January, 1990, to 1 January, 2013. Our specific focus is on comparing models with and without spillover effects from the S\&P 500 index, while for the S\&P 500 index we investigate whether crashes in the index are provoked by crashes in the NASDAQ. Within sample, a Lagrange Multiplier test rejectes the absence of crossexcitation except for the cross-excitation to the NASDAQ. Residual analysis confirms the adequacy of the models.

Out-of-sample, we evaluate the forecasts of the probability of an extreme and the Value-atRisk forecasts at the $95 \%$ and $99 \%$ confidence level from the models from 1 January, 2013, to 1 July, 2015. The probability scores and the adjusted Mean Squared Prediction Error test show that the Hawkes models with spillover effects and predictable event sizes provide the most accurate forecasts of the probability of an extreme event for all series except for positive extremes in the bond market. The incorporation of triggering effect that depends on the size of past events, does not necessarily lead to more accurate probability predictions. The results for the VaR forecasts also indicate the added value of cross-excitation. 
Our results show more generally that extreme events in financial markets should not be modelled or forecast in isolation but in joint models. Though our attention is restricted to crossexcitation from one process to another, our results provide an incentive for further research into multivariate Hawkes models.

\section{References}

[1] Aït-Sahalia, Y., Laeven, R. J., and Pelizzon, L. (2014). Mutual excitation in Eurozone sovereign CDS. Journal of Econometrics, 183(2): 151-167.

[2] Aït-Sahalia, Y., Cacho-Diaz, J., and Laeven, R. (2015). Modeling financial contagion using mutually exciting jump processes. Journal of Financial Economics, 117(3): 585-606.

[3] Bae, K. H., and Karolyi, G. A. (1994). Good news, bad news and international spillovers of stock return volatility between Japan and the US. Pacific-Basin Finance Journal, 2(4): 405-438.

[4] Bae, K. H., Karolyi, G. A., and Stulz, R. M. (2003). A new approach to measuring financial contagion. Review of Financial Studies, 16(3): 717-763.

[5] Bauwens, L., and Hautsch, N. (2009). Modeling financial high frequency data using point processes. In: Handbook of Financial Time Series, 953-979. Springer Berlin Heidelberg.

[6] Bekaert, G., Ehrmann, M., Fratzscher, M., and Mehl, A. J. (2011). Global crises and equity market contagion. NBER Working paper.

[7] Berkowitz, J., Christoffersen, P., and Pelletier, D. (2011). Evaluating value-at-risk models with desk-level data. Management Science, 57(12): 2213-2227.

[8] Booth, G. G., Martikainen, T., and Tse, Y. (1997). Price and volatility spillovers in Scandinavian stock markets. Journal of Banking \& Finance, 21(6): 811-823.

[9] Bormetti, G., Calcagnile, L., Treccani, M., Corsi, F., Marmi, S., and Lillo, F. (2013). Modeling systemic cojumps with Hawkes factor models. arXiv preprint arXiv:1301.6141.

[10] Bowsher, C. G. (2002). Modelling security market events in continuous time: Intensity based, multivariate point process models. Economics Discussion Paper No. 2002-W22, Nuffield College, Oxford.

[11] Bowsher, C. G. (2007). Modeling security market events in continuous time: Intensity based, multivariate point process models. Journal of Econometrics, 141(2): 876-912. 
[12] Breusch, T. S., and Pagan, A. R. (1980). The Lagrange multiplier test and its applications to model specification in econometrics. The Review of Economic Studies, 47(1): 239-253.

[13] Byström, H. N. (2004). Managing extreme risks in tranquil and volatile markets using conditional extreme value theory. International Review of Financial Analysis, 13(2): 133-152.

[14] Chavez-Demoulin, V., Davison, A., and McNeill, A. (2005). Estimating value-at-risk: a point process approach. Quantitative Finance 5(2): 227-234.

[15] Chavez-Demoulin, V., and McGill, J. (2012). High-frequency financial data modeling using Hawkes processes. Journal of Banking \& Finance, 36(12): 3415-3426.

[16] Christoffersen, P. F. (1998). Evaluating interval forecasts. International Economic Review, 39(4): 841-862.

[17] Clark, T., and West, K., (2007). Approximately normal tests for equal predictive accuracy in nested models. Journal of Econometrics, 138(1): 291-311.

[18] Connolly, R. A., and Wang, F. A. (2003). International equity market comovements: Economic fundamentals or contagion? Pacific-Basin Finance Journal, 11(1): 23-43

[19] Diebold, F., and Mariano, R. (1995). Comparing predictive accuracy. Journal of Business and Economic Statistics, 13(3): 253-265.

[20] Engle, R. F. (1982). A general approach to Lagrange multiplier model diagnostics. Journal of Econometrics, 20(1): 83-104.

[21] Engle, R. F., and Manganelli, S. (2004). CAViaR: Conditional autoregressive value at risk by regression quantiles. Journal of Business \& Economic Statistics, 22(4): 367-381.

[22] Embrechts, P., Liniger, T., and Lin, L. (2011). Multivariate Hawkes processes: an application to financial data. Journal of Applied Probability, 48A: 367-378.

[23] Eun, C. S., and Shim, S. (1989). International transmission of stock market movements. Journal of Financial and Quantitative Analysis, 24(2): 241-256.

[24] Fischer, K. P., and Palasvirta, A. P. (1990). High road to a global marketplace: the international transmission of stock market fluctuations. Financial Review, 25(3): 371-394.

[25] Giacomini, R., and Komunjer, I. (2005). Evaluation and combination of conditional quantile forecasts. Journal of Business \& Economic Statistics, 23(4): 416-431. 
[26] Gonzalo, J., and Olmo, J. (2004). Which extreme values are really extreme? Journal of Financial Econometrics, 2(3): 349-369.

[27] Gourieroux, C., Monfort, A., and Renne, J.P. (2014). Pricing default events: Surprise, exogeneity and contagion. Journal of Econometrics, 182(2): 397-411.

[28] Gresnigt, F., Kole, E. and Franses, P. H. (2015a). Interpreting financial market crashes as earthquakes: A new early warning system for medium term crashes. Journal of Banking and Finance, 56(1): 123-139.

[29] Gresnigt, F., Kole, E., and Franses, P. H. (2015b). Specification testing in Hawkes models. TI working paper (No. TI 15-086/III).

[30] Grothe, O., Korniichuk, V., and Manner, H., (2014). Modeling multivariate extreme events using self-exciting point processes. Journal of Econometrics, 182(2): 269-289.

[31] Hamao, Y., Masulis, R. W., and Ng, V. (1990). Correlations in price changes and volatility across international stock markets. Review of Financial studies, 3(2): 281-307.

[32] Hamilton, J. D. (1996). Specification testing in Markov-switching time-series models. Journal of Econometrics, 70(1): 127-157.

[33] Hartmann, P., Straetmans, S., and De Vries, C. G. (2004). Asset market linkages in crisis periods. Review of Economics and Statistics, 86(1): 313-326.

[34] Herrera, R. and Schipp, B. (2009). Self-exciting extreme value models for stock market crashes. In: Statistical Inference, Econometric Analysis and Matrix Algebra, 209-231. Physica-Verlag HD.

[35] Hewlett, P. (2006). Clustering of order arrivals, price impact and trade path optimization. In: Workshop on Financial Modeling with Jump processes, Ecole Polytechnique.

[36] Kanas, A. (1998). Volatility spillovers across equity markets: European evidence. Applied Financial Economics, 8(3): 2450-256.

[37] King, M. A., and Wadhwani, S. (1990). Transmission of volatility between stock markets. Review of Financial Studies, 3(1): 5-33.

[38] Koutmos, G., and Booth, G. G. (1995). Asymmetric volatility transmission in international stock markets. Journal of International Money and Finance, 14(6): 747-762: 5-33. 
[39] Lin, W. L., Engle, R. F., and Ito, T. (1994). Do bulls and bears move across borders? International transmission of stock returns and volatility. Review of Financial Studies, 7(3): 507-538.

[40] Longin, F., and Solnik, B. (2001). Extreme correlation of international equity markets. The Journal of Finance, 56(2): 649-676.

[41] Ogata, Y. (1988). Statistical models for earthquake occurrences and residual analysis for point processes. Journal of the American Statistical Association, 83(401): 9-27.

[42] Ogata, Y. (1998). Space-time point-process models for earthquake occurrences. Annals of the Institute of Statistical Mathematics, 50(2): 379-402.

[43] Pickands III, J. (1975). Statistical inference using extreme order statistics. Annals of Statistics, 3(1): 119-131.

[44] Poon, S. H., Rockinger, M., and Tawn, J. (2003). Modelling extreme-value dependence in international stock markets. Statistica Sinica, 13(4): 929-954.

[45] Poon, S. H., Rockinger, M., and Tawn, J. (2004). Extreme value dependence in financial markets: Diagnostics, models, and financial implications. Review of Financial Studies, 17(2): $581-610$.

[46] Scarrott, C., and MacDonald, A. (2012). A review of extreme value threshold estimation and uncertainty quantification. REVSTAT - Statistical Journal 10(1): 33-60.

[47] Sun, W., Rachev, S., Fabozzi, F. J., and Kalev, P. S. (2009). A new approach to modeling co-movement of international equity markets: evidence of unconditional copula-based simulation of tail dependence. Empirical economics, 36(1): 201-229. 


\section{List of Figures}

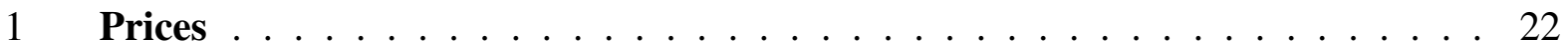

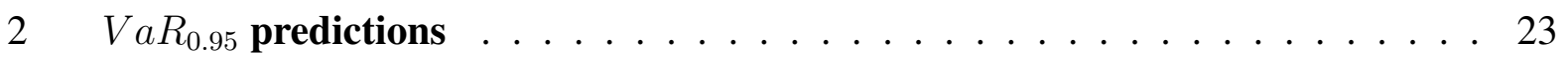




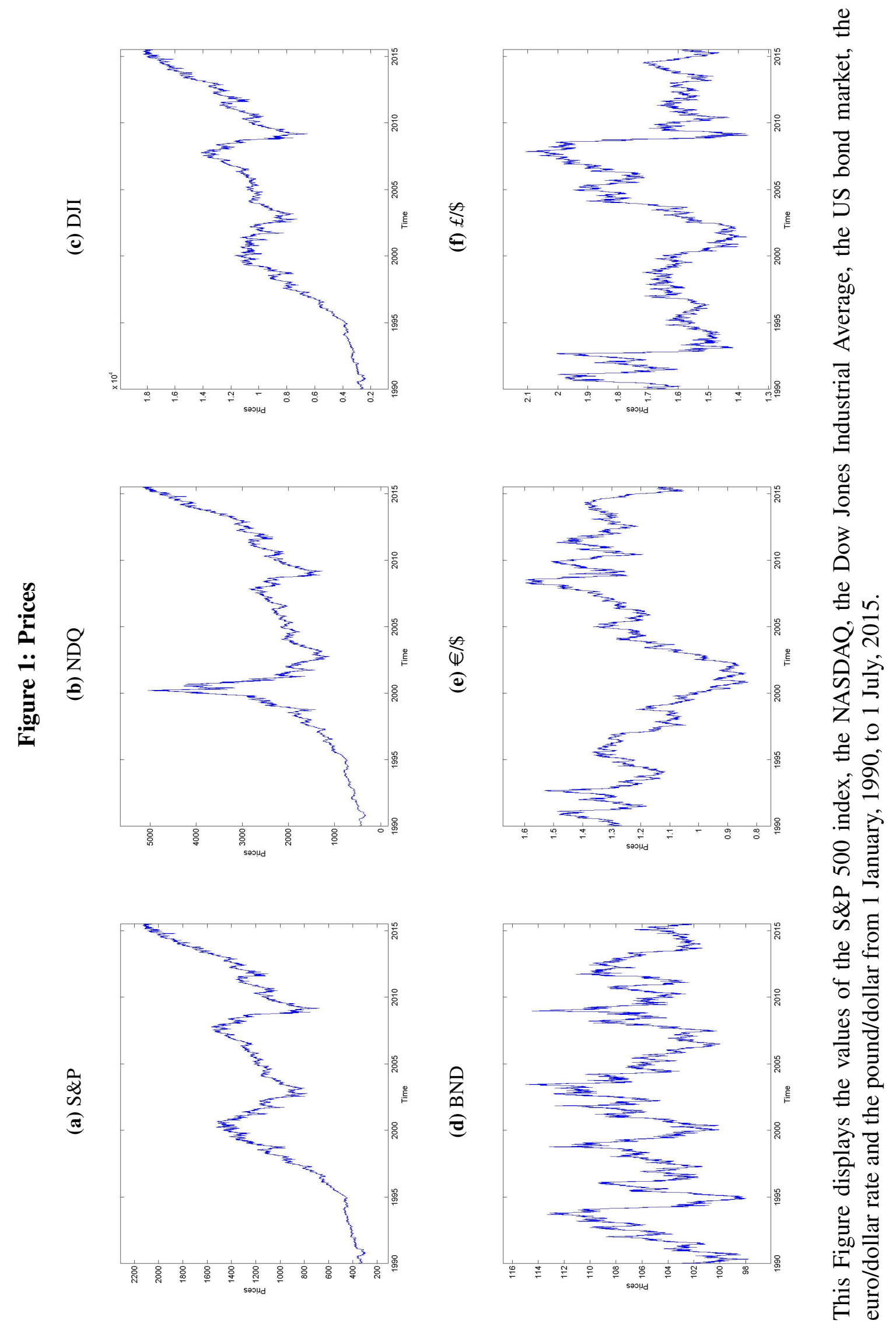




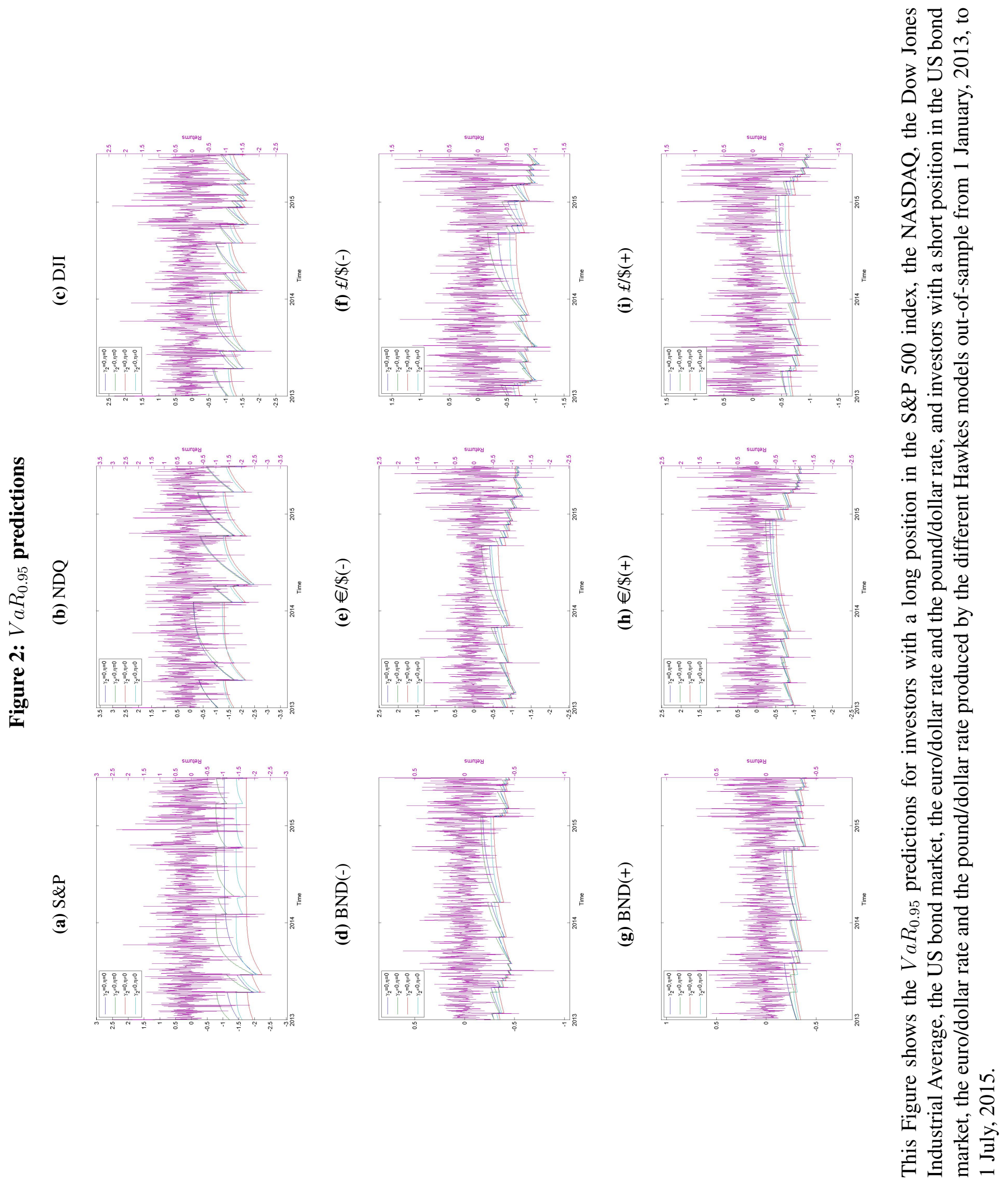




\section{List of Tables}

1 Parameter estimates . . . . . . . . . . . . . . . . . . . . . 25

2 LM statistics dependence test $\ldots \ldots \ldots 26$

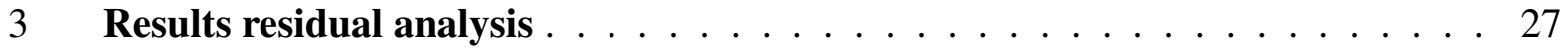

4 Probability scores . . . . . . . . . . . . . . . . . . . . . . . 28

5 Adjusted MSPE statistics testing cross-excitation . . . . . . . . . . . . . . . . . . 29

6 Adjusted MSPE statistics testing the influence on/of sizes $\ldots \ldots \ldots$

$7 \quad L R_{u c}, L R_{i n d}$ and $L R_{c c}$ test statistics $V a R_{0.95} \ldots \ldots \ldots \ldots \ldots \ldots \ldots \ldots$

$8 \quad L R_{u c}, L R_{\text {ind }}$ and $L R_{c c}$ test statistics $V a R_{0.99} \ldots \ldots \ldots \ldots \ldots \ldots \ldots \ldots \ldots$

9 DQ test statistics . . . . . . . . . . . . . . . . . . 33

10 DM statistics testing cross-excitation . . . . . . . . . . . . . . . . . . 34

11 DM statistics testing the influence on/of sizes $\ldots \ldots \ldots 35$ 





Table 2: LM statistics dependence test

\begin{tabular}{rll|cccc}
\hline & & & \multicolumn{3}{|c}{$\alpha=0$} & \multicolumn{2}{c}{$\alpha \neq 0$} \\
& & & $\eta=0$ & $\eta \neq 0$ & $\eta=0$ & $\eta \neq 0$ \\
\hline S\&P & vs. & NDQ & 3.50 & 4.33 & 4.10 & 5.46 \\
NDQ & vs. & S\&P & 0.86 & 0.99 & 0.52 & 0.62 \\
DJI & vs. & S\&P & 4.44 & 5.94 & 1.33 & 1.62 \\
BND(-) & vs. & S\&P & 33.98 & 34.49 & 34.38 & 34.51 \\
$€ / \$(-)$ & vs. & S\&P & 17.56 & 18.20 & 15.18 & 15.00 \\
$£ / \$(-)$ & vs. & S\&P & 13.60 & 13.89 & 12.33 & 12.18 \\
BND(+) & vs. & S\&P & 10.73 & 11.20 & 11.06 & 11.56 \\
$€ / \$(+)$ & vs. & S\&P & 17.07 & 17.69 & 14.15 & 14.09 \\
$£ / \$(+)$ & vs. & S\&P & 15.92 & 16.22 & 8.42 & 7.87 \\
\hline
\end{tabular}

LM statistics for testing the null-hypothesis of no spillover effects from the NASDAQ to the S\&P 500 index and from the S\&P 500 index to the NASDAQ, the DJI index, the US bond index, the euro/dollar rate and the pound/dollar rate. The tests are applied to extreme returns above the 94.29\% quantile over a period that starts at January 1, 1990 and ends at January 1, 2013. In the Hawkes models with the parameter restriction $\alpha=0$, the magnitude of events have no influence on the triggering subsequent events. In the Hawkes models with the parameter restriction $\eta=0$, the history of the events has no influence on the magnitude of subsequent events. The critical value corresponding to a $5 \%$ significance level is equal to 3.84 . 
Table 3: Results residual analysis

\begin{tabular}{c|ccccccccc}
\hline & \multicolumn{9}{|c}{$\alpha=0$} \\
& \multicolumn{2}{|c}{$\eta=0$} & \multicolumn{2}{c}{$\eta \neq 0$} & \multicolumn{3}{c}{$\alpha \neq 0$} \\
& $\gamma_{2}=0$ & $\gamma_{2} \neq 0$ & $\gamma_{2}=0$ & $\gamma_{2} \neq 0$ & $\gamma_{2}=0$ & $\gamma_{2} \neq 0$ & $\gamma_{2}=0$ & $\gamma_{2} \neq 0$ \\
\hline S\&P & 0.595 & 0.102 & 0.641 & 0.076 & 0.385 & 0.226 & 0.294 & 0.227 \\
NDQ & 0.453 & 0.171 & 0.596 & 0.179 & 0.332 & 0.183 & 0.491 & 0.262 \\
DJI & 0.519 & 0.167 & 0.547 & 0.323 & 0.230 & 0.134 & 0.405 & 0.262 \\
BND(-) & 0.566 & 0.028 & 0.576 & 0.038 & 0.683 & 0.028 & 0.717 & 0.034 \\
$€ / \$(-)$ & 0.662 & 0.192 & 0.654 & 0.237 & 0.708 & 0.212 & 0.686 & 0.213 \\
$£ / \$(-)$ & 0.390 & 0.119 & 0.426 & 0.159 & 0.305 & 0.074 & 0.365 & 0.120 \\
BND(+) & 0.411 & 0.038 & 0.428 & 0.040 & 0.411 & 0.038 & 0.416 & 0.040 \\
$€ / \$(+)$ & 0.648 & 0.157 & 0.663 & 0.113 & 0.641 & 0.168 & 0.647 & 0.120 \\
$£ / \$(+)$ & 0.432 & 0.126 & 0.453 & 0.105 & 0.480 & 0.203 & 0.487 & 0.165 \\
\hline
\end{tabular}

The $p$-values of the Kolmogorov-Smirnov tests performed on the transformed times $\left\{\tau_{i}\right\}$ specified by the models. The null-hypothesis of the test states that the transformed times $\left\{\tau_{i}\right\}$ are distributed according to a homogeneous Poisson process with intensity 1 . The models are applied to extreme negative returns in three US stock market indices and extreme negative and positive returns in the US bond market and two exchange rates over a period that starts at January 1, 1990 and ends at January 1, 2013. The threshold above which extremes are considered, is set at $94.29 \%$ quantile employing a procedure of Gonzalo and Olmo (2004). For the S\&P 500 index, $\gamma_{2}$ controls the spillover effect from crashes in the NASDAQ, while for the DJI index, the US bond index, the euro/dollar rate and the pound/dollar this parameter controls the spillover effect from crashes in the S\&P 500. In the Hawkes models with the parameter restriction $\alpha=0$, the magnitude of events have no influence on the triggering subsequent events. In the Hawkes models with the parameter restriction $\eta=0$, the history of the events has no influence on the magnitude of subsequent events. 
Table 4: Probability scores

\begin{tabular}{|c|c|c|c|c|c|c|c|c|c|}
\hline & \multicolumn{4}{|c|}{$\alpha=0$} & \multicolumn{4}{|c|}{$\alpha \neq 0$} \\
\hline & & \multicolumn{2}{|c|}{$\eta=0$} & \multicolumn{2}{|c|}{$\eta \neq 0$} & \multicolumn{2}{|c|}{$\eta=0$} & \multicolumn{2}{|c|}{$\eta \neq 0$} \\
\hline & & $\gamma_{2}=0$ & $\gamma_{2} \neq 0$ & $\gamma_{2}=0$ & $\gamma_{2} \neq 0$ & $\gamma_{2}=0$ & $\gamma_{2} \neq 0$ & $\gamma_{2}=0$ & $\gamma_{2} \neq 0$ \\
\hline Series & Scores & & & & & & & & \\
\hline \multirow[t]{2}{*}{$\mathrm{S} \& \mathrm{P}$} & QPS & 0.0002 & 0.0001 & 0.0003 & 0.0001 & 0.0002 & 0.0001 & 0.0003 & 0.0001 \\
\hline & LPS & 0.0092 & 0.0066 & 0.0099 & 0.0068 & 0.0093 & 0.0064 & 0.0104 & 0.0066 \\
\hline \multirow[t]{2}{*}{ NDQ } & QPS & 0.0005 & 0.0005 & 0.0006 & 0.0006 & 0.0004 & 0.0004 & 0.0005 & 0.0005 \\
\hline & LPS & 0.0137 & 0.0134 & 0.0144 & 0.0141 & 0.0128 & 0.0127 & 0.0135 & 0.0133 \\
\hline \multirow[t]{2}{*}{ DJI } & QPS & 0.0015 & 0.0013 & 0.0017 & 0.0015 & 0.0014 & 0.0013 & 0.0015 & 0.0014 \\
\hline & LPS & 0.0254 & 0.0240 & 0.0265 & 0.0252 & 0.0242 & 0.0238 & 0.0253 & 0.0249 \\
\hline \multirow[t]{2}{*}{ BND(-) } & QPS & 0.0016 & 0.0013 & 0.0016 & 0.0013 & 0.0015 & 0.0013 & 0.0015 & 0.0013 \\
\hline & LPS & 0.0250 & 0.0221 & 0.0252 & 0.0224 & 0.0248 & 0.0221 & 0.0252 & 0.0225 \\
\hline \multirow[t]{2}{*}{$€ / \$(-)$} & QPS & 0.0039 & 0.0030 & 0.0039 & 0.0031 & 0.0037 & 0.0030 & 0.0036 & 0.0030 \\
\hline & LPS & 0.0368 & 0.0313 & 0.0367 & 0.0318 & 0.0361 & 0.0315 & 0.0361 & 0.0324 \\
\hline \multirow[t]{2}{*}{$£ / \$(-)$} & QPS & 0.0026 & 0.0020 & 0.0028 & 0.0023 & 0.0022 & 0.0018 & 0.0024 & 0.0020 \\
\hline & LPS & 0.0323 & 0.0276 & 0.0335 & 0.0295 & 0.0306 & 0.0269 & 0.0317 & 0.0286 \\
\hline \multirow[t]{2}{*}{$\mathrm{BND}(+)$} & QPS & 0.0008 & 0.0009 & 0.0008 & 0.0010 & 0.0008 & 0.0009 & 0.0008 & 0.0010 \\
\hline & LPS & 0.0191 & 0.0202 & 0.0193 & 0.0211 & 0.0191 & 0.0202 & 0.0192 & 0.0211 \\
\hline \multirow[t]{2}{*}{$€ / \$(+)$} & QPS & 0.0021 & 0.0017 & 0.0021 & 0.0017 & 0.0020 & 0.0017 & 0.0021 & 0.0017 \\
\hline & LPS & 0.0268 & 0.0239 & 0.0271 & 0.0240 & 0.0267 & 0.0240 & 0.0271 & 0.0243 \\
\hline \multirow[t]{2}{*}{$£ / \$(+)$} & QPS & 0.0014 & 0.0011 & 0.0014 & 0.0012 & 0.0013 & 0.0011 & 0.0014 & 0.0012 \\
\hline & LPS & 0.0227 & 0.0203 & 0.0233 & 0.0208 & 0.0233 & 0.0210 & 0.0241 & 0.0219 \\
\hline
\end{tabular}

QPS and LPS of the probability predictions of crashes the next day in the S\&P 500 index, the NASDAQ and the Dow Jones Industrial Average, and crashes and booms in the US bond index, the euro/dollar rate and the pond/dollar rate out-of-sample from 1 January, 2013, to 1 July, 2015. The QPS and LPS are given in (18) and (19). The QPS and LPS range respectively from 0 to 1 and from 0 to $\infty$, with 0 indicating perfect accuracy. 
Table 5: Adjusted MSPE statistics testing cross-excitation

\begin{tabular}{c|cccc}
\hline & \multicolumn{2}{|c}{$\alpha=0$} & \multicolumn{2}{c}{$\alpha \neq 0$} \\
& $\eta=0$ & $\eta \neq 0$ & $\eta=0$ & $\eta \neq 0$ \\
\hline S\&P & 13.57 & 13.55 & 15.70 & 20.25 \\
NDQ & 6.53 & 5.79 & 5.99 & 5.35 \\
DJI & 8.66 & 7.81 & 6.67 & 6.24 \\
BND(-) & 16.36 & 15.92 & 13.15 & 14.48 \\
$€ / \$(-)$ & 14.33 & 14.96 & 14.36 & 14.76 \\
$£ / \$(-)$ & 21.56 & 20.88 & 28.93 & 29.24 \\
BND $(+)$ & -3.24 & -8.19 & -3.24 & -8.75 \\
$€ / \$(+)$ & 10.99 & 10.33 & 11.08 & 10.26 \\
$£ / \$(+)$ & 11.26 & 10.60 & 21.34 & 19.38 \\
\hline
\end{tabular}

Adjusted MSPE statistics comparing probability predictions of models with and without crossexcitation. The models predict the probability of crashes the next day in the S\&P 500 index, the NASDAQ and the Dow Jones Industrial Average, and crashes and booms in the US bond index, the euro/dollar rate and the pond/dollar rate out-of-sample from 1 January, 2013, to 1 July, 2015. We use the adjusted MSPE test of Clark and West (2007) as we compare nested models. The critical value corresponding to a $5 \%$ significance level is equal to 1.64 . 
Table 6: Adjusted MSPE statistics testing the influence on/of sizes

\begin{tabular}{c|cccccccc}
\hline & \multicolumn{3}{|c}{$\eta(\alpha=0)$} & \multicolumn{2}{c}{$\alpha(\eta=0)$} & \multicolumn{2}{c}{$\eta(\alpha \neq 0)$} & \multicolumn{2}{c}{$\alpha(\eta \neq 0)$} \\
& $\gamma_{2}=0$ & $\gamma_{2} \neq 0$ & $\gamma_{2}=0$ & $\gamma_{2} \neq 0$ & $\gamma_{2}=0$ & $\gamma_{2} \neq 0$ & $\gamma_{2}=0$ & $\gamma_{2} \neq 0$ \\
\hline S\&P & 7.94 & 3.50 & -3.64 & -5.21 & 17.71 & 3.51 & -0.29 & -4.51 \\
NDQ & 5.51 & 5.42 & -9.87 & -10.24 & 5.55 & 5.50 & -8.31 & -8.54 \\
DJI & 6.95 & 7.76 & -11.97 & -4.48 & 7.02 & 7.67 & -10.63 & -4.60 \\
$\mathrm{BND}(-)$ & 9.23 & 9.75 & -6.61 & -3.48 & 9.22 & 7.32 & -4.14 & -1.09 \\
$\in / \$(-)$ & 8.32 & 10.46 & -6.48 & -1.80 & 5.31 & 7.65 & -6.29 & -1.94 \\
$£ / \$(-)$ & 8.63 & 10.50 & -12.76 & -11.89 & 8.73 & 10.88 & -11.43 & -10.70 \\
$\mathrm{BND}(+)$ & 10.51 & 26.05 & -10.70 & -36.46 & 10.02 & 26.05 & -7.41 & -31.49 \\
$\in / \$(+)$ & 6.40 & 2.82 & -4.70 & 0.48 & 6.67 & 3.73 & -3.99 & 0.96 \\
$£ / \$(+)$ & 9.54 & 10.53 & -3.51 & -2.40 & 20.55 & 23.31 & -3.16 & -1.51 \\
\hline
\end{tabular}

Adjusted MSPE statistics comparing probability predictions of models with and without influence of the the history of the events on the magnitude of subsequent events $(\eta)$ and models with and without influence of the magnitude of events triggering subsequent events $(\alpha)$. The models predict the probability of crashes the next day in the S\&P 500 index, the NASDAQ and the Dow Jones Industrial Average, and crashes and booms in the US bond index, the euro/dollar rate and the pond/dollar rate out-of-sample from 1 January, 2013, to 1 July, 2015. We use the adjusted MSPE test of Clark and West (2007) as we compare nested models. The critical value corresponding to a $5 \%$ significance level is equal to 1.64 . 
Table 7: $L R_{u c}, L R_{i n d}$ and $L R_{c c}$ test statistics $V a R_{0.95}$

\begin{tabular}{|c|c|c|c|c|c|c|c|c|c|}
\hline & \multicolumn{4}{|c|}{$\alpha=0$} & \multicolumn{4}{|c|}{$\alpha \neq 0$} \\
\hline & & \multicolumn{2}{|c|}{$\eta=0$} & \multicolumn{2}{|c|}{$\eta \neq 0$} & \multicolumn{2}{|c|}{$\eta=0$} & \multicolumn{2}{|c|}{$\eta \neq 0$} \\
\hline & & $\gamma_{2}=0$ & $\gamma_{2} \neq 0$ & $\gamma_{2}=0$ & $\gamma_{2} \neq 0$ & $\gamma_{2}=0$ & $\gamma_{2} \neq 0$ & $\gamma_{2}=0$ & $\gamma_{2} \neq 0$ \\
\hline \multirow[t]{3}{*}{ S\&P } & $L R_{u c}$ & 0.01 & 14.69 & 22.38 & 6.98 & 0.01 & 11.39 & 22.38 & 6.98 \\
\hline & $L R_{\text {ind }}$ & 2.68 & 1.06 & 0.31 & 1.14 & 3.02 & 0.71 & 0.31 & 1.14 \\
\hline & $L R_{c c}$ & 2.69 & 15.76 & 22.69 & 8.12 & 3.03 & 12.10 & 22.69 & 8.12 \\
\hline \multirow[t]{3}{*}{ NDQ } & $L R_{u c}$ & 87.29 & 94.53 & 1.07 & 0.72 & 84.93 & 94.53 & 0.72 & 0.72 \\
\hline & $L R_{\text {ind }}$ & 5.39 & 9.76 & 0.01 & 0.04 & 4.57 & 8.13 & 0.04 & 0.04 \\
\hline & $L R_{c c}$ & 92.68 & 104.29 & 1.09 & 0.76 & 89.50 & 102.66 & 0.76 & 0.76 \\
\hline \multirow[t]{3}{*}{ DJI } & $L R_{u c}$ & 5.93 & 5.93 & 2.02 & 0.72 & 3.80 & 5.93 & 1.51 & 0.72 \\
\hline & $L R_{\text {ind }}$ & 0.12 & 0.79 & 0.00 & 0.49 & 0.37 & 0.79 & 0.00 & 0.49 \\
\hline & $L R_{c c}$ & 6.05 & 6.71 & 2.02 & 1.20 & 4.17 & 6.71 & 1.51 & 1.20 \\
\hline \multirow[t]{3}{*}{$\mathrm{BND}(-)$} & $L R_{u c}$ & 2.63 & 5.17 & 2.02 & 0.22 & 1.65 & 5.17 & 2.02 & 0.43 \\
\hline & $L R_{\text {ind }}$ & 0.03 & 0.02 & 2.00 & 2.90 & 1.23 & 0.02 & 2.00 & 2.71 \\
\hline & $L R_{c c}$ & 2.66 & 5.19 & 4.02 & 3.12 & 2.89 & 5.19 & 4.02 & 3.14 \\
\hline \multirow[t]{3}{*}{$€ / \$(-)$} & $L R_{u c}$ & 0.90 & 26.63 & 0.06 & 0.18 & 0.36 & 9.40 & 0.06 & 0.36 \\
\hline & $L R_{\text {ind }}$ & 0.28 & 1.10 & 0.03 & 0.64 & 0.00 & 0.28 & 0.03 & 0.50 \\
\hline & $L R_{c c}$ & 1.18 & 27.73 & 0.09 & 0.82 & 0.36 & 9.68 & 0.09 & 0.87 \\
\hline \multirow[t]{3}{*}{$£ / \$(-)$} & $L R_{u c}$ & 5.93 & 31.25 & 1.07 & 1.65 & 6.73 & 20.96 & 1.07 & 0.90 \\
\hline & $L R_{\text {ind }}$ & 0.12 & 2.37 & 2.44 & 0.13 & 0.07 & 2.02 & 2.44 & 0.28 \\
\hline & $L R_{c c}$ & 6.05 & 33.62 & 3.51 & 1.78 & 6.79 & 22.98 & 3.51 & 1.18 \\
\hline \multirow[t]{3}{*}{$\mathrm{BND}(+)$} & $L R_{u c}$ & 10.37 & 4.46 & 1.51 & 0.22 & 10.37 & 4.46 & 1.07 & 0.22 \\
\hline & $L R_{\text {ind }}$ & 0.19 & 0.00 & 0.78 & 1.60 & 0.19 & 0.00 & 0.62 & 1.60 \\
\hline & $L R_{c c}$ & 10.57 & 4.47 & 2.28 & 1.83 & 10.57 & 4.47 & 1.69 & 1.83 \\
\hline \multirow[t]{3}{*}{$€ / \$(+)$} & $L R_{u c}$ & 2.63 & 12.45 & 0.43 & 1.25 & 3.19 & 11.39 & 0.43 & 0.06 \\
\hline & $L R_{\text {ind }}$ & 1.83 & 2.77 & 1.86 & 2.69 & 1.58 & 3.10 & 1.86 & 0.79 \\
\hline & $L R_{c c}$ & 4.45 & 15.23 & 2.29 & 3.94 & 4.77 & 14.50 & 2.29 & 0.86 \\
\hline \multirow[t]{3}{*}{$£ / \$(+)$} & $L R_{u c}$ & 0.60 & 3.19 & 2.02 & 0.72 & 0.01 & 0.60 & 2.62 & 0.22 \\
\hline & $L R_{\text {ind }}$ & 0.01 & 0.49 & 2.00 & 0.04 & 0.35 & 0.39 & 1.84 & 0.27 \\
\hline & $L R_{c c}$ & 0.61 & 3.68 & 4.02 & 0.76 & 0.35 & 0.99 & 4.46 & 0.49 \\
\hline
\end{tabular}

Statistics for the unconditional coverage, independence and conditional coverage tests on the VaR predictions at a $95 \%$ confidence level produced by the different Hawkes models. The models predict the maximum of returns associated with a 95\% confidence level the next day in the S\&P 500 index, the NASDAQ and the Dow Jones Industrial Average, and crashes and booms in the US bond index, the euro/dollar rate and the pond/dollar rate out-of-sample from 1 January, 2013, to 1 July, 2015. The critical value corresponding to a $5 \%$ significance level is equal to 3.84 for the unconditional coverage test and the independence test (asymptotic $\chi^{2}(1)$-distribution) and 5.99 for the conditional coverage test (asymptotic $\chi^{2}(2)$-distribution). 
Table 8: $L R_{u c}, L R_{i n d}$ and $L R_{c c}$ test statistics $V a R_{0.99}$

\begin{tabular}{|c|c|c|c|c|c|c|c|c|c|}
\hline & \multicolumn{4}{|c|}{$\alpha=0$} & \multicolumn{4}{|c|}{$\alpha \neq 0$} \\
\hline & & \multicolumn{2}{|c|}{$\eta=0$} & \multicolumn{2}{|c|}{$\eta \neq 0$} & \multicolumn{2}{|c|}{$\eta=0$} & \multicolumn{2}{|c|}{$\eta \neq 0$} \\
\hline & & $\gamma_{2}=0$ & $\gamma_{2} \neq 0$ & $\gamma_{2}=0$ & $\gamma_{2} \neq 0$ & $\gamma_{2}=0$ & $\gamma_{2} \neq 0$ & $\gamma_{2}=0$ & $\gamma_{2} \neq 0$ \\
\hline \multirow[t]{3}{*}{ S\&P } & $L R_{u c}$ & 0.32 & 2.58 & 2.40 & 0.03 & 0.32 & 3.73 & 2.40 & 0.03 \\
\hline & $L R_{\text {ind }}$ & 0.20 & 0.38 & 0.03 & 0.15 & 0.20 & 0.45 & 0.03 & 0.15 \\
\hline & $L R_{c c}$ & 0.52 & 2.96 & 2.43 & 0.19 & 0.52 & 4.18 & 2.43 & 0.19 \\
\hline \multirow[t]{3}{*}{ NDQ } & $L R_{u c}$ & 2.58 & 2.58 & 0.85 & 0.03 & 2.58 & 2.58 & 0.85 & 0.32 \\
\hline & $L R_{\text {ind }}$ & 1.86 & 1.86 & 0.25 & 0.15 & 1.86 & 1.86 & 0.25 & 0.20 \\
\hline & $L R_{c c}$ & 4.44 & 4.44 & 1.10 & 0.19 & 4.44 & 4.44 & 1.10 & 0.52 \\
\hline \multirow[t]{3}{*}{ DJI } & $L R_{u c}$ & 1.14 & 1.14 & 0.04 & 0.39 & 1.14 & 1.14 & 0.04 & 0.39 \\
\hline & $L R_{\text {ind }}$ & 0.05 & 0.05 & 0.11 & 0.08 & 0.05 & 0.05 & 0.11 & 0.08 \\
\hline & $L R_{c c}$ & 1.19 & 1.19 & 0.15 & 0.47 & 1.19 & 1.19 & 0.15 & 0.47 \\
\hline \multirow[t]{3}{*}{$\mathrm{BND}(-)$} & $L R_{u c}$ & 0.04 & 0.03 & 0.03 & 0.03 & 0.39 & 0.03 & 0.03 & 0.03 \\
\hline & $L R_{\text {ind }}$ & 0.11 & 0.15 & 0.15 & 0.15 & 0.08 & 0.15 & 0.15 & 0.15 \\
\hline & $L R_{c c}$ & 0.15 & 0.19 & 0.19 & 0.19 & 0.47 & 0.19 & 0.19 & 0.19 \\
\hline \multirow[t]{3}{*}{$€ / \$(-)$} & $L R_{u c}$ & 0.04 & 0.03 & 5.05 & 0.85 & 0.04 & 0.32 & 3.73 & 0.85 \\
\hline & $L R_{\text {ind }}$ & 0.11 & 0.15 & 1.31 & 0.25 & 0.11 & 0.20 & 1.57 & 0.25 \\
\hline & $L R_{c c}$ & 0.15 & 0.19 & 6.35 & 1.10 & 0.15 & 0.52 & 5.29 & 1.10 \\
\hline \multirow[t]{3}{*}{$£ / \$(-)$} & $L R_{u c}$ & 2.40 & 0.39 & 2.58 & 0.04 & 2.40 & 1.14 & 2.58 & 0.03 \\
\hline & $L R_{\text {ind }}$ & 0.03 & 0.08 & 1.86 & 0.11 & 0.03 & 0.05 & 1.86 & 0.15 \\
\hline & $L R_{c c}$ & 2.43 & 0.47 & 4.44 & 0.15 & 2.43 & 1.19 & 4.44 & 0.19 \\
\hline \multirow[t]{3}{*}{$\mathrm{BND}(+)$} & $L R_{u c}$ & 0.04 & 1.14 & 0.85 & 1.14 & 0.04 & 1.14 & 0.85 & 1.14 \\
\hline & $L R_{\text {ind }}$ & 0.11 & 0.05 & 0.25 & 0.05 & 0.11 & 0.05 & 0.25 & 0.05 \\
\hline & $L R_{c c}$ & 0.15 & 1.19 & 1.10 & 1.19 & 0.15 & 1.19 & 1.10 & 1.19 \\
\hline \multirow[t]{3}{*}{$€ / \$(+)$} & $L R_{u c}$ & 0.03 & 0.32 & 3.73 & 2.58 & 0.03 & 0.85 & 2.58 & 2.58 \\
\hline & $L R_{\text {ind }}$ & 0.15 & 0.20 & 0.45 & 0.38 & 0.15 & 0.25 & 0.38 & 0.38 \\
\hline & $L R_{c c}$ & 0.19 & 0.52 & 4.18 & 2.96 & 0.19 & 1.10 & 2.96 & 2.96 \\
\hline \multirow[t]{3}{*}{$£ / \$(+)$} & $L R_{u c}$ & 0.04 & 0.03 & 0.32 & 0.03 & 0.04 & 0.03 & 0.32 & 0.32 \\
\hline & $L R_{\text {ind }}$ & 0.11 & 0.15 & 0.20 & 0.15 & 0.11 & 0.15 & 0.20 & 0.20 \\
\hline & $L R_{c c}$ & 0.15 & 0.19 & 0.52 & 0.19 & 0.15 & 0.19 & 0.52 & 0.52 \\
\hline
\end{tabular}

Statistics for the unconditional coverage, independence and conditional coverage tests on the VaR predictions at a $99 \%$ confidence level produced by the different Hawkes models. The models predict the maximum of returns associated with a $99 \%$ confidence level the next day in the S\&P 500 index, the NASDAQ and the Dow Jones Industrial Average, and crashes and booms in the US bond index, the euro/dollar rate and the pond/dollar rate out-of-sample from 1 January, 2013, to 1 July, 2015. The critical value corresponding to a $5 \%$ significance level is equal to 3.84 for the unconditional coverage test and the independence test (asymptotic $\chi^{2}(1)$-distribution) and 5.99 for the conditional coverage test (asymptotic $\chi^{2}(2)$-distribution). 
Table 9: DQ test statistics

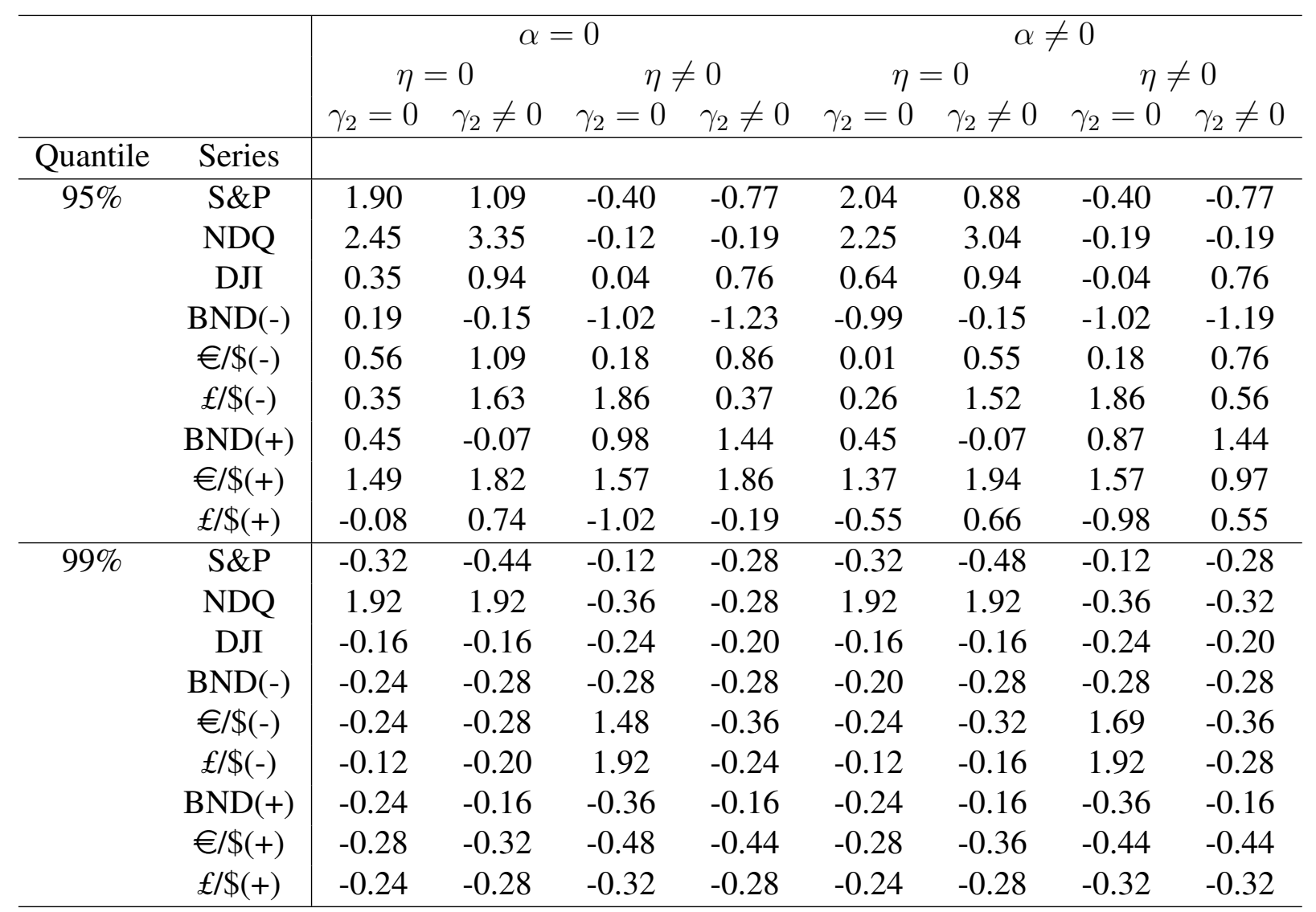

Statistics for the Dynamic Quantile test on the VaR predictions at a 95\% and 99\% confidence level produced by the different Hawkes models. The models predict the maximum of returns associated with a 95\% confidence level the next day in the S\&P 500 index, the NASDAQ and the Dow Jones Industrial Average, and crashes and booms in the US bond index, the euro/dollar rate and the pond/dollar rate out-of-sample from 1 January, 2013, to 1 July, 2015. The critical value corresponding to a $5 \%$ significance level is equal to \pm 1.96 . 


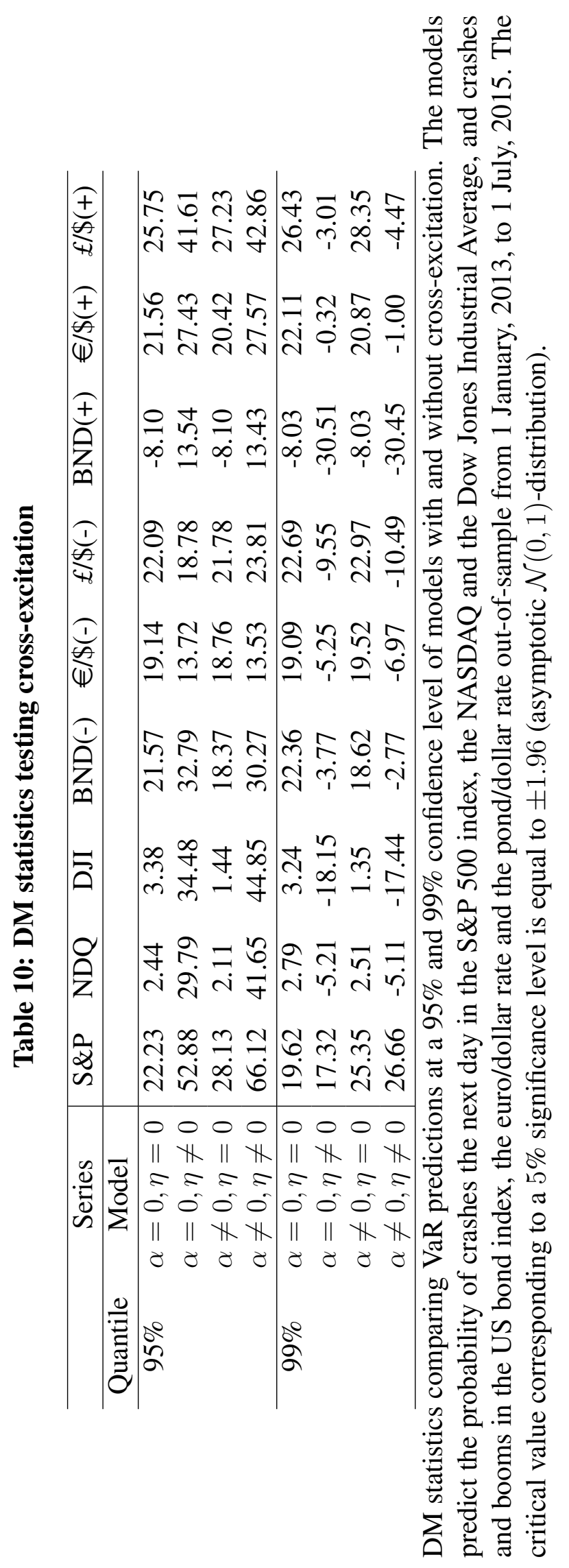


Table 11: DM statistics testing the influence on/of sizes

\begin{tabular}{cc|cccccccc}
\hline & & \multicolumn{3}{|c}{$\eta(\alpha=0)$} & \multicolumn{2}{c}{$\alpha(\eta=0)$} & \multicolumn{2}{c}{$\eta(\alpha \neq 0)$} & \multicolumn{2}{c}{$\alpha(\eta \neq 0)$} \\
& & $\gamma_{2}=0$ & $\gamma_{2} \neq 0$ & $\gamma_{2}=0$ & $\gamma_{2} \neq 0$ & $\gamma_{2}=0$ & $\gamma_{2} \neq 0$ & $\gamma_{2}=0$ & $\gamma_{2} \neq 0$ \\
\hline Quantile & Series & & & & & & & & \\
\hline $95 \%$ & S\&P & -65.03 & -56.95 & -8.38 & 1.39 & -65.70 & -59.48 & 32.53 & 56.22 \\
& NDQ & -42.93 & -40.17 & 11.32 & 10.98 & -46.60 & -43.07 & 24.36 & 20.87 \\
& DJI & -26.77 & -28.71 & 8.58 & 4.30 & -30.16 & -28.55 & 33.04 & 12.43 \\
& BND(-) & -23.01 & -21.80 & -1.17 & -0.33 & -23.63 & -21.03 & -5.32 & -7.71 \\
& $€ / \$(-)$ & -11.60 & -15.87 & -2.52 & -6.12 & -12.15 & -15.30 & -0.57 & -5.65 \\
& $£ / \$(-)$ & -15.79 & -16.66 & 2.45 & -2.46 & -18.91 & -17.68 & 7.69 & 3.36 \\
& BND(+) & -39.91 & -33.92 & 5.80 & 32.92 & -39.49 & -33.92 & 14.52 & 39.49 \\
& $€ / \$(+)$ & -19.96 & -17.81 & -5.23 & -8.22 & -20.19 & -17.29 & -5.52 & -8.04 \\
& $£ / \$(+)$ & -27.67 & -24.69 & -8.91 & -10.39 & -31.52 & -26.61 & -8.70 & -9.08 \\
\hline $99 \%$ & S\&P & -7.01 & -18.64 & -8.87 & 0.97 & -8.19 & -19.10 & -22.57 & 5.67 \\
& NDQ & 4.40 & 3.27 & 11.03 & 10.43 & 3.09 & 2.03 & 6.36 & 6.68 \\
& DJI & 22.63 & 29.71 & 8.27 & 4.29 & 23.31 & 28.33 & -14.31 & -0.16 \\
& BND(-) & 19.17 & 13.23 & -1.70 & -4.47 & 18.63 & 10.98 & -3.64 & -4.02 \\
& $€ / \$(-)$ & 20.08 & 5.43 & -2.35 & -6.30 & 20.59 & 4.70 & -8.12 & -5.66 \\
& $£ / \$(-)$ & 22.96 & 9.67 & 2.30 & -3.10 & 25.45 & 13.89 & -12.33 & 0.77 \\
& BND(+) & 20.27 & 22.86 & 8.33 & 31.77 & 20.07 & 22.86 & -7.10 & 23.64 \\
& $\in / \$(+)$ & 15.78 & 5.88 & -5.41 & -8.59 & 15.62 & 4.29 & -7.82 & -9.69 \\
& $\mathbb{1} \$(+)$ & 16.28 & 9.83 & -9.00 & -10.54 & 21.12 & 13.84 & -9.54 & -9.13 \\
\hline
\end{tabular}

DM statistics comparing VaR predictions at the 95-99\% confidence level of models with and without influence of the the history of the events on the magnitude of subsequent events $(\eta)$ and models with and without influence of the magnitude of events triggering subsequent events $(\alpha)$. The models predict the probability of crashes the next day in the S\&P 500 index, the NASDAQ and the Dow Jones Industrial Average, and crashes and booms in the US bond index, the euro/dollar rate and the pond/dollar rate out-of-sample from 1 January, 2013, to 1 July, 2015. The critical value corresponding to a $5 \%$ significance level is equal to \pm 1.96 (asymptotic $\mathcal{N}(0,1)$-distribution). 